\title{
Implementierung eines Betriebskonzeptes in einer HNO-Klinik im Rahmen der SARS-CoV-2-Pandemie
}

\section{Implementation of an operational concept in an ENT clinic in the context of the SARS-CoV-2 pandemic}

Autoren

Roxanne Weiss ${ }^{1}$, Andreas Loth ${ }^{1}$, Daniela Guderian¹, Marc Diensthuber ${ }^{1}$, Volkhard Kempf ${ }^{2}$, Daniel Hack ${ }^{2}$, Sabine Wicker ${ }^{3}$, Sandra Ciesek ${ }^{4}$, Jürgen Graf ${ }^{5}$, Timo Stöver ${ }^{1}$, Martin Leinung ${ }^{1}$

Institute

1 Klinik für Hals-Nasen-Ohrenheilkunde, Universitätsklinikum Frankfurt, Frankfurt am Main, Germany

2 Institut für Medizinische Mikrobiologie und Krankenhaushygiene, Universitäres Zentrum für Infektionskrankheiten, Hessisches universitäres Kompetenzzentrum Krankenhaushygiene, Universitätsklinikum Frankfurt, Frankfurt am Main, Germany

3 Betriebsärztlicher Dienst, Universitätsklinikum Frankfurt, Frankfurt am Main, Germany

4 Institut für Medizinische Virologie, Universitätsklinikum Frankfurt, Frankfurt am Main, Germany

5 Ärztlicher Direktor und Vorstandsvorsitzender, Universitätsklinikum Frankfurt, Frankfurt am Main, Germany

Schlüsselwörter

SARS-CoV-2, Betriebskonzept, Patientensicherheit, Mitarbeiterschutz, Pandemie

Key words

SARS-CoV-2, operational concept, patient safety, employee protection, pandemic

eingereicht 27.04 .2020

akzeptiert 28.04.2020

Bibliografie

DOI https://doi.org/10.1055/a-1174-0234

Online-Publikation: 28.5.2020

Laryngo-Rhino-Otol 2020; 99: 552-560

(c) Georg Thieme Verlag KG, Stuttgart · New York ISSN 0935-8943

Korrespondenzadresse

Dr. Roxanne Weiss

Klinik für Hals-Nasen-Ohrenheilkunde, Universitätsklinikum Frankfurt, Theodor-Stern-Kai 7, 60590 Frankfurt am Main,

Germany

Tel.: ++49/69/63015674

Fax: ++49/69/630180473

roxanne.weiss@kgu.de

\section{ZUSAMMENFASSUNG}

Die SARS-CoV-2-Pandemie stellt das gesamte medizinische Versorgungssystem vor große Herausforderungen. Gerade in universitären Einrichtungen als Maximalversorger ist eine höhere Exposition zu potenziell infektiösen Patienten oder tatsächlichen COVID-19-Kranken zu erwarten. Es musste kurzfristig ein Betriebskonzept entwickelt werden, das den aktuellen Hygiene-Empfehlungen des Robert Koch-Instituts (RKI), den Empfehlungen der führenden Fachgesellschaften und dem daraus abgeleiteten internen Hygieneplan entsprach. Hierbei sind die Patientensicherheit und der Mitarbeiterschutz gleichermaßen zu beachten.

In Zusammenarbeit mit dem Institut für Medizinische Mikrobiologie und Krankenhaushygiene sowie dem Betriebsärztlichen Dienst wurden Maßnahmen festgelegt, um trotz begrenzter diagnostischer und Ausrüstungsressourcen Lösungen zu erarbeiten, um die COVID-19-Übertragungsgefahr für Untersucher und Patienten zu minimieren. Hierzu wurde ein Betriebskonzept erarbeitet, das aus verschiedenen Einzelmaßnahmen bestand, wie z. B. der Reduktion der ambulanten Patientenbehandlung auf Notfälle, lebensbedrohliche Erkrankungen und dringliche Nachsorgen, eine Doppeltriage von Patienten sowie die Einführung von Behandlungsteams. Das neue erarbeitete Betriebskonzept konnte erfolgreich innerhalb weniger Tage umgesetzt werden. Nach ersten Erfahrungen aus dem Realbetrieb und mehreren „Hygienebegehungen“ waren lediglich geringfügige Nachbesserungen des Konzeptes erforderlich. Alle Maßnahmen wurden schriftlich im klinikinternen Qualitätshandbuch dokumentiert und sind allen Mitarbeitern zugänglich. Da es sich bei der SARS-CoV-2Pandemie um einen dynamischen Prozess mit regelmäßiger Änderung des Entwicklungs- und Informationsstandes handelt, wird das Betriebskonzept regelmäßig neu auf Gültigkeit überprüft und ggf. angepasst.

\section{ABSTRACT}

The SARS-CoV-2 pandemic poses major challenges for the entire medical care system. Especially in university institutions as maximum care providers, a higher exposure to potentially infectious patients or actual COVID-19 patients is to be expected. In a short period of time, an operational concept had to be developed regarding the current hygiene recommendations 
of the Robert Koch Institute (RKI), the leading medical societies and the internal hospital hygiene plan. Here, patient safety and employee protection are equally important.

In cooperation with the Institute for Medical Microbiology and Hospital Hygiene and the occupational medical service, interventions were defined to develop solutions to minimize the COVID-19 transmission risk for examiners and patients despite limited diagnostic and equipment resources. For this purpose, an operational concept was developed, consisting of various individual actions, e. g. the reduction of outpatient treatment to emergencies, life-threatening diseases and ur- gent aftercare, a double triage of patients and the introduction of treatment teams.

The newly developed operational concept was successfully implemented within a few days. After the initial rollout and several "hygiene inspections" only minor improvements to the concept were necessary. All measures were documented in the internal quality handbook and are accessible to all employees. Since the SARS-CoV-2 pandemic is a dynamic process with regular changes in the development and information status, the operational concept is regularly reviewed for validity and adjusted as necessary.

\section{Einleitung}

Das neue Coronavirus (initial als „2019-nCoV“ benannt, aktuell SARS-CoV-2) ist ein bis dahin unbekanntes Virus aus der Familie der Coronaviridae, das im Dezember 2019 als Auslöser von Lungenerkrankungen identifiziert wurde. Vielfach verläuft die Infektion klinisch inapparent oder als banaler Infekt der oberen Atemwege $[1,2]$. Die schwerere klinische Verlaufsform, das schwere akute Atemwegssyndrom (Severe Acute Respiratory Syndrome, SARS), kann als atypische Pneumonie mit den Hauptsymptomen Husten, Atembeschwerden und Fieberanstieg imponieren und wird als COVID-19 (englisch für „corona virus disease 2019“) bezeichnet. Mittlerweile ist die Verbreitung von SARS-CoV-2 so weit fortgeschritten, dass die WHO im März 2020 die bisherige Epidemie offiziell zu einer Pandemie erklärt hat [3]. Die Pandemie stellt die gesamte Welt vor eine enorme soziale, wirtschaftliche und vor allem medizinische Herausforderung.

Bei infizierten Patienten lässt sich regelhaft eine hohe Viruslast in den oberen und unteren Atemwegen nachweisen [4, 5]. Viren wie SARS-CoV-2 werden nicht vereinzelt in der Luft übertragen, sondern sind in Tröpfchen eingeschlossen. Pro Atemzug stößt der Mensch 1000-50 000 Tröpfchen aus, beim Husten sind die Tröpfchen um ein 10-Faches größer. Virenhaltige Aerosole können durch Manipulationen und invasive Maßnahmen an betroffenen Schleimhäuten entstehen und halten sich länger in der Luft. Dadurch ergibt sich eine erhöhte Infektiosität [6-8]. Mitarbeiter im Gesundheitssystem sind hier aufgrund der physischen Nähe zu Patienten vermutlich besonders gefährdet. So zeigte eine Untersuchung des Chinese Center for Disease Control and Prevention, dass von allen positiv Getesteten 3,8\% (1716 von 44672) Angehörige des Gesundheitssystems waren. 63 \% (1080 von 1716) davon kamen aus der am stärksten betroffenen Region Wuhan [9]. Auch das RKI ermittelte für die Kalenderwoche 15 einen Anteil von $6 \%$ an medizinischem Personal unter den registrierten COVID-19-Fällen. Da allerdings bei $40 \%$ der Fälle noch Angaben zur Beschäftigung fehlen, liegt der tatsächliche Anteil möglicherweise höher [10]. Insbesondere HNO-Ärzte scheinen ebenfalls aufgrund der Lokalisation ihrer Tätigkeiten am Patienten einer erhöhten Exposition und Viruslast ausgesetzt zu sein [11]. Die Pandemie stellt dadurch plötzlich stark veränderte Ansprüche an die HNO-ärztlichen Untersuchungs- und Behandlungsabläufe und macht tiefgreifende Anpassungen der etablierten klinischen Pro- zesse erforderlich, um die Sicherheit des Personals und der Patienten zu gewährleisten. Ziel dieser Arbeit ist die Darstellung der Entwicklung und Umsetzung eines an die SARS-CoV-2-Pandemie angepassten Betriebskonzeptes einer universitären HNO-Klinik.

\section{Material und Methoden}

Der Standort ist eine universitäre Einrichtung und somit ein Krankenhaus der hochschulmedizinischen Maximalversorgung. Zeitgleich zur notwendigen Reorganisation der Arbeitsabläufe der Klinik für Hals-Nasen-Ohrenheilkunde wurde klinikweit, entsprechend der Empfehlung des RKI, eine organisatorische und räumliche Trennung in einen COVID-positiven und einen COVID-negativen Bereich vollzogen [12]. In beiden Bereichen werden zusätzlich Räume vorgehalten für Patienten mit unklarem COVID-Status (sogenannte „graue Zone“). Die Räumlichkeiten der HNO-Klinik sind dem COVID-negativen Bereich zugeordnet. Eine konsiliarische Mitbetreuung von COVID-positiven Patienten außerhalb der HNO-Klinik ist ebenfalls erforderlich.

Initial wurden mit dem Institut für Medizinische Mikrobiologie und Krankenhaushygiene die besonderen Untersuchungs- und Behandlungsbedingungen im Kopf-Hals-Bereich definiert und die Eckpfeiler des Konzepts festgelegt. Diese beinhalteten:

- die Wegeführung der Patienten,

- die ausschließliche Durchführung der körperlichen HNOUntersuchung in voller persönlicher Schutzausrüstung (Voll-PSA) aufgrund der erhöhten Exposition,

- die konsequente Minimierung der Anzahl und der Expositionszeit pro Patient sowie

- die Vermeidung von operativen Eingriffen ohne COVID-negativen Status.

Hierbei war es erforderlich, sowohl die Empfehlungen medizinischer Fachgesellschaften [13], des Robert Koch-Instituts (RKI) als auch die internen Hygienerichtlinien zu beachten. Außerdem galt es, die notwendige Einsparung knapper Ressourcen (MundNasen-Schutz (MNS), FFP2/3-Masken, SARS-CoV-2-Tests) zu berücksichtigen ( $\triangleright$ Abb. $\mathbf{1})$.

Die daraus resultierende erste Version des Betriebskonzeptes wurde in einem iterativen Prozess von allen Beteiligten ausgearbeitet. In Aspekte des Mitarbeiterschutzes und der Arbeitssicherheit wurde der Betriebsärztliche Dienst eingebunden, mit dem 


\begin{tabular}{|c|c|c|c|c|c|c|}
\hline \multirow{5}{*}{ 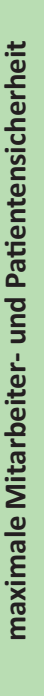 } & \multirow{5}{*}{$\begin{array}{c}\text { Herausforderungen } \\
\text { Mitarbeiterschutz } \\
\text { Patientenschutz } \\
\text { Infektionsprävention } \\
\text { Limitationen } \\
\text { Ressourcenknappheit } \\
\text { persönliche Schutzausrüstung } \\
\text { Testkapazitäten } \\
\text { bauliche Infrastruktur } \\
\text { Personal } \\
\\
\text { Vorgaben } \\
\text { Fachgesellschaften } \\
\text { Robert Koch-Institut } \\
\text { interner Hygieneplan } \\
\text { bestehendes Betriebskonzept }\end{array}$} & Prozess & & Ziele & & Konzept \\
\hline & & Patientenannahme & 0 & $\begin{array}{l}\text { Kontrolle der } \\
\text { Patientenzugänge } \\
\text { Reduktion der Patientenzahl }\end{array}$ & $\begin{array}{l}0 \\
0 \\
0 \\
0 \\
0\end{array}$ & $\begin{array}{l}\text { Limitierung Gebäudezugang durch Sicherheitsdienst } \\
\text { Schließung der Nebeneingänge } \\
\text { Begrenzung der Begleitpersonen } \\
\text { Beschränkung auf Not-und Eilfälle, wenige Nachsorgen } \\
\text { Doppeltriage der Patienten }\end{array}$ \\
\hline & & $\begin{array}{c}\text { Ambulante } \\
\text { Patientenbehandlung }\end{array}$ & 0 & $\begin{array}{l}\text { Reduktion } \\
\text { Patientenkontaktzeit }\end{array}$ & $\begin{array}{l}0 \\
0\end{array}$ & $\begin{array}{l}\text { Untersuchung in Voll-PSA } \\
\text { Streng symptomorientierte Untersuchung } \\
\text { Bilden von Behandlungsteams aus: } 1 \text { Untersucher, } 1 \text { Dokumentar, } \\
1 \text { Assistenzperson } \\
\text { Räumliche Trennung der Untersuchung und Dokumentation } \\
\text { Reduktion der Verbrauchsmaterialien im Untersuchungsraum auf } \\
\text { ein Minimum }\end{array}$ \\
\hline & & $\begin{array}{c}\text { Stationäre } \\
\text { Patientenbehandlung }\end{array}$ & 0 & $\begin{array}{l}\text { Reduktion Anzahl } \\
\text { stationärer Patienten } \\
\text { Isolation bis COVID-negativ }\end{array}$ & $\begin{array}{l}0 \\
0 \\
0\end{array}$ & $\begin{array}{l}\text { Strenge Indikationsstellung zur stationären Aufnahme } \\
\text { Abstrich bei allen Aufnahmen } \\
\text { Einrichtung einer "grauen Zone“ (Isolationszimmer) bis zum } \\
\text { Vorliegen des Abstrichergebnisses }\end{array}$ \\
\hline & & $\begin{array}{c}\text { Operative } \\
\text { Patientenbehandlung }\end{array}$ & 0 & $\begin{array}{l}\text { Reduktion der Operationen } \\
\text { auf ein Minimum } \\
\text { Erhöhung Sicherheit OP- } \\
\text { Team (alle Berufsgruppen) }\end{array}$ & $\begin{array}{l}0 \\
0 \\
0 \\
0\end{array}$ & $\begin{array}{l}\text { Strenge Indikationsstellung } \\
\text { Einteilung in elektiv, dringlich, Eil- und Notoperationen (vgl. Abb. 5) } \\
\text { Präoperative Abstrichkontrolle } \\
\text { Wahl der Schutzausrüstung und Ort der Operation nach } \\
\text { Abstrichergebnis } \\
\text { Vermeidung Aerosol-bildender Verfahren }\end{array}$ \\
\hline
\end{tabular}

- Abb. 1 Übersicht der Herausforderungen, Ziele und Umsetzungsstrategien bei der Erstellung des Betriebskonzeptes.

das finale Konzept ebenfalls abgestimmt wurde. Entsprechend der klinisch relevanten Arbeitsabläufe wurde das Betriebskonzept in die 4 organisatorischen Bereiche Patientenannahme, ambulante, stationäre und operative Patientenbehandlung gegliedert.

\section{Ergebnisse}

Seit dem 16.03.2020 werden am Standort ausschließlich Notfälle, lebensbedrohliche Erkrankungen und nachstationäre Patienten behandelt. Bis zum Vorliegen eines negativen SARS-CoV2-Abstrich-ergebnisses wird jeder ambulant oder stationär behandelte Patient als potenziell infektiös betrachtet, auch wenn zum Zeitpunkt der Implementierung des Konzeptes die Durchseuchung der Bevölkerung - selbst unter Berücksichtigung einer Dunkelziffer - noch relativ gering war.

\section{Patientenannahme}

Entscheidende Faktoren zur Strukturierung der Patientenannahme sind die Kontrolle des Patientenzulaufs und die Reduktion der Patientenzahl, um das Übertragungsrisiko zu minimieren. Dazu wurde der Zugang zum Gebäude limitiert. Nebeneingänge wurden (in der gesamten Einrichtung) verschlossen und Mitarbeiter eines neu beauftragten Sicherheitsdienstes kontrollieren den Zugang zur HNO-Klinik anhand von Mitarbeiterausweisen, Identifikationsarmbändern stationärer Patienten und Terminbescheinigungen ambulanter Patienten. Zur Reduktion des Individualkontaktes wurde zudem eine Beschränkung begleitender Personen beschlossen. Erforderlichenfalls dürfen die Patienten von maximal einem Elternteil, Betreuer oder Übersetzer begleitet werden. Weitere Begleiter werden gebeten, vor dem Gebäude zu warten ( Abb. 2).

Der Zutritt zum Gebäude wird nur nach telefonischer Rücksprache des Sicherheitsdienstes und Freigabe durch die Mitarbeiter der Hochschulambulanz (HSA) gewährt. Dabei ist die Anzahl der Patienten im Wartebereich auf maximal 7 Personen limitiert, um Hygieneempfehlungen im Hinblick auf einen Sicherheitsabstand von mindestens 1,5 m zwischen den Wartenden zu gewährleisten.

In der Anmeldung der Hochschulambulanz (HSA) werden neu eingetroffene Patienten von administrativen Mitarbeitern mit Tages-MNS (Mund-Nase-Schutz) befragt, ob sie einem der 3 obengenannten Kriterien (Notfall, schwere Erkrankung oder Nachsorge) entsprechen. Anschließend werden die patientenbezogenen Daten in das Krankenhausinformationssystem (KIS) aufgenommen (Stammdatenerfassung).

Im Anschluss erfolgt durch einen erfahrenen Assistenzarzt eine Doppeltriage zur Klärung, ob der klinische Verdacht auf eine SARS-CoV-2-Infektion vorliegt und ob das HNO-Krankheitsbild eine akute Behandlung erfordert. Die Triage auf SARS-CoV-2 ist dabei eng an die Empfehlungen des RKI angelehnt [14]. Je nach Ergebnis der Doppeltriage wird der Patient ambulant behandelt, an das örtliche Corona-Testcenter weiterverwiesen oder die

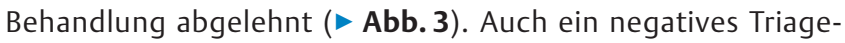
Ergebnis mit Abweisung des Patienten wird aus ökonomischen und medikolegalen Gründen dokumentiert.

\section{Ambulante Patientenbehandlung}

Primäres Organisationsziel in der ambulanten Patientenbehandlung ist die Reduktion der Kontaktzeit zum Patienten im Sinne des Mitarbeiterschutzes. Dazu erfolgt die Patientenbehandlung in Teams zu je 3 Personen ( $\triangleright$ Abb. 4):

- einem Untersucher (HNO-Arzt mit der größten klinischen Erfahrung),

- einem Dokumentar (jüngerer HNO-Assistent, der aber mit der Terminologie, den Abläufen sowie dem Umgang mit dem Krankenhausinformationssystem (KIS) vertraut ist) sowie - einer Assistenzkraft. 


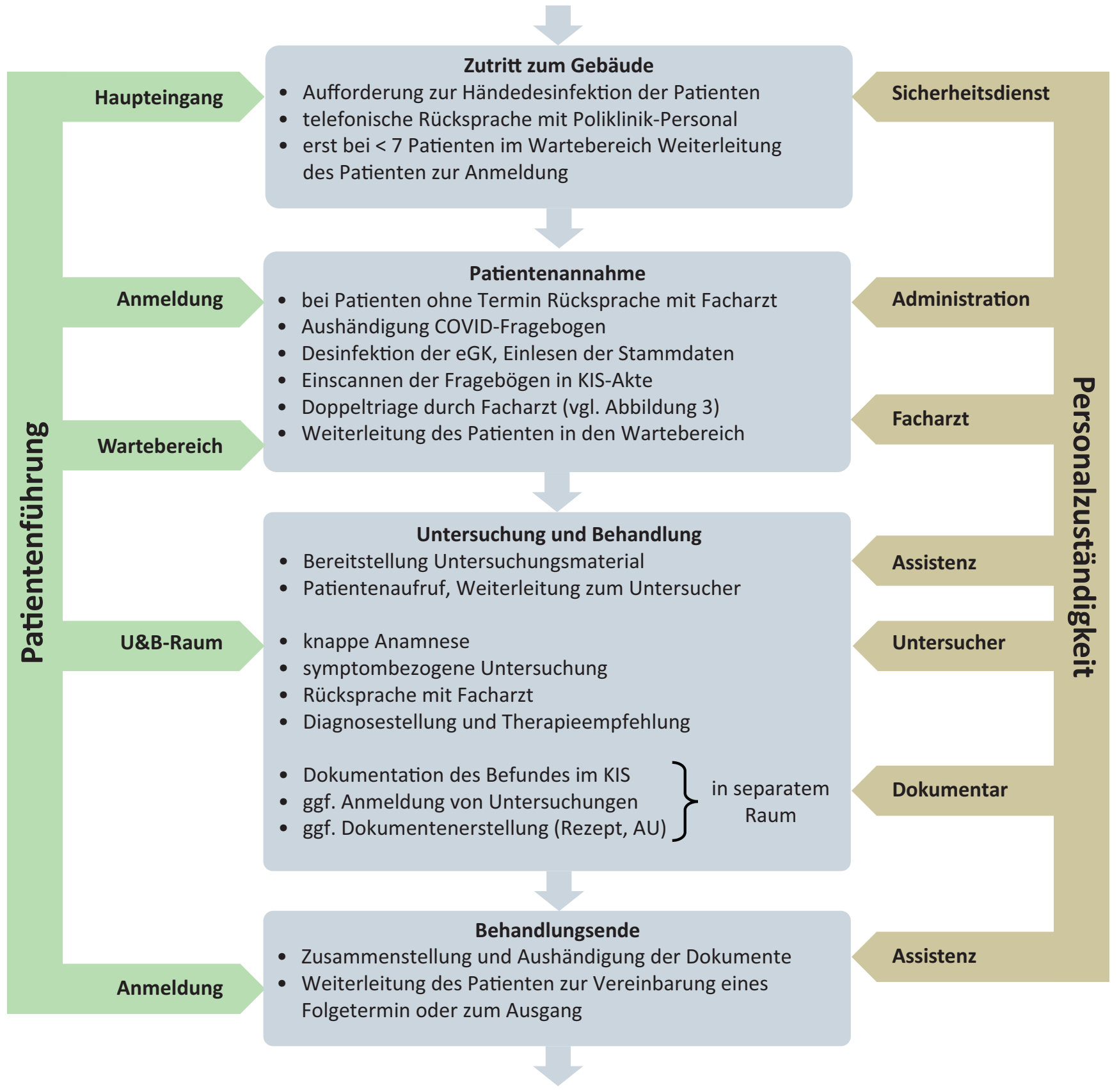

- Abb. 2 Flussschema der ambulanten Patientenbehandlung. Links aufgetragen der Patientenfluss (grün) innerhalb des Behandlungsprozesses bis zum Verlassen der Ambulanz. Rechts die entsprechende Personalzuständigkeit innerhalb der Teilbereiche (grau). U\&B= Untersuchung und Behandlung; eGK = elektronische Gesundheitskarte; KIS = Krankenhausinformationssystem; AU = Arbeitsunfähigkeitsbescheinigung.

Je nach Verfügbarkeit kann die Assistenz auch eine Pflegekraft, ein Student im praktischen Jahr oder eine studentische Hilfskraft sein.

Außerdem wurden 2 Untersuchungs- und Behandlungsräume der Hochschulambulanz (HSA) für die Patientenbehandlung ausgewiesen. Einer dieser Räume ist für Rettungsdienstannahmen oder stationäre Liegendpatienten bettengängig. Die Untersuchungseinheiten sind aufgrund des potenziellen Kontaminationsrisikos von Instrumenten und Verbandsmaterial entleert. Stündlich erfolgt eine Desinfektion (begrenzt viruzid) aller besonders häufig kontaktierten Oberflächen (z. B. Türklinken, Armlehnen etc.) sowie alle 4 Stunden eine Desinfektion aller exponierten Flächen im Untersuchungsraum (begrenzt viruzid). Ebenso erfolgt die regelmäßige Lüftung der Räume, um einen entsprechenden Luftwechsel zu gewährleisten. Die technische Ausstattung der Untersuchungseinheiten wurde für die Untersuchung potenziell infektiöser Patienten um Endoskopkameras und Bildschirme ergänzt, um die Notwendigkeit körperlicher Annäherung zwischen Arzt und Patient zu minimieren. Dies ermöglicht auch bei Bedarf eine Befunddemonstration über den Videobildschirm an den Oberarzt. 


\begin{tabular}{|c|c|c|c|}
\hline & & \multicolumn{2}{|c|}{ klinischer V.a. SARS-CoV-2? } \\
\hline & & 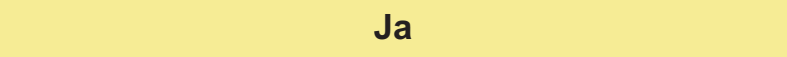 & Nein \\
\hline \multirow{2}{*}{$\begin{array}{l}\text { HNO- } \\
\text { Krankheitsbild } \\
\text { behandlungs- } \\
\text { würdig? }\end{array}$} & Ja & $\begin{array}{c}\text { Patient erhält chirurgischen MNS, wird gebeten, bis zum } \\
\text { Aufrufen vor dem Gebäude zu warten und später direkt } \\
\text { in die Untersuchungskabine verbracht. Nach der } \\
\text { Behandlung Flächendesinfektion der Kabine, } \\
\text { Handschuhwechsel und - im Falle einer Kontamination } \\
\text { - Kittelwechsel und Entsorgung gemäß Hygieneplan der } \\
\text { Einrichtung). }\end{array}$ & $\begin{array}{l}\text { Patient wird ohne weitere } \\
\text { Schutzmaßnahmen in den } \\
\text { Wartebereich der HSA* } \\
\text { gebeten, sofern dort eine } \\
\text { Maximalzahl von } 7 \\
\text { wartenden Patienten nicht } \\
\text { überschritten wird.* }\end{array}$ \\
\hline & Nein & $\begin{array}{l}\text { Empfehlung zur Vorstellung im örtlichen Corona- } \\
\text { Testcenter }\end{array}$ & $\begin{array}{l}\text { Dokumentation des } \\
\text { Triageergebnisses im } \\
\text { KIS *** und Ablehnung der } \\
\text { Patientenbehandlung }\end{array}$ \\
\hline
\end{tabular}

- Abb. 3 Schema der Doppeltriage. Die Symptome einer COVID-19-Erkrankung wurden mit einem Fragebogen erhoben. * HSA=Hochschulambulanz; ** Bei zu hohem Patientenandrang wird der Zugang zum Gebäude durch die Sicherheitskräfte am Eingang limitiert;

*** KIS= Krankenhausinformationssystem.

Der Untersucher tritt als einzige Person in direkten Kontakt zum Patienten und ist aufgrund potenzieller Infektiosität in VollPSA eingekleidet (FFP2/3-Maske, Schutzbrille, Kopfhaube, wasserabweisender Kittel und Handschuhe). Er trägt chirurgische Handschuhe unter den Einmalhandschuhen, die zum Patientenschutz nach jeder abgeschlossenen Behandlung gewechselt werden. Der Untersucher verbleibt in der Untersuchungskabine und verlässt diese nur zu Pausenzeiten. Seine Aufgabe besteht in der Erhebung der Anamnese und der streng symptombezogenen körperlichen Untersuchung des Patienten.

Über eine Telefonverbindung mit Freisprechanlage verfolgt der Dokumentar das Gespräch mit, führt die Dokumentation in der elektronischen Patientenakte durch und veranlasst notwendige administrative Maßnahmen (Rezepterstellung etc.). Auf einem Monitor im Untersuchungsraum sieht der Untersucher einen gespiegelten Bildschirm des Dokumentars, um Eintragungen zu kontrollieren und ggf. zu korrigieren.

Die für die Behandlung benötigten Instrumente werden nach Bedarf durch die Assistenz hereingereicht. Diese ruft auch die Patienten auf oder geleitet sie wieder hinaus. Auch andere Funktionen wie die Ausgabe von Dokumenten oder die Überwachung des Wartebereichs gehören zu ihren Aufgaben. Vor einer Pause oder Personalwechsel des Untersuchers wird die Voll-PSA abgelegt und entsprechend der im Vorfeld einrichtungsweit erfolgten Schulungen und gemäß Hygieneplan als Festmüll entsorgt.

\section{Stationäre Patientenbehandlung}

Im stationären Bereich gilt es, COVID-negative Patienten von neuen Patienten mit unklarem Infektionsstatus zu trennen. Daher werden alle stationären Patienten bei der Aufnahme abgestrichen. Bis zum negativen Nachweis auf SARS-CoV-2 werden die Patienten räumlich isoliert, die Patientenzimmer werden als sog. „graue Zone“ ausgewiesen. Die Patienten sind angehalten, ihr Zimmer bis zur Freigabe nicht zu verlassen. Es erfolgt eine Ein- fachbelegung eines Zimmers, um bei einem zufällig nachgewiesenen Fall keine Kontaktpatienten generiert zu haben. Bei einem Massenandrang von Patienten wäre eine Kohortierung jedoch möglich. Auch postoperativ zu betreuende Patienten ohne COVID-Abstrichergebnis verbleiben zunächst bis zum Vorliegen des Laborbefundes in der grauen Zone.

Da auch einfache HNO-ärztliche Tätigkeiten wie das Absaugen der Nasenhaupthöhlen oder Tracheostomata mit einem erhöhten Risiko für die Behandler assoziiert zu sein scheinen, werden diese Maßnahmen auch bei stationären Patienten mit unklarem COVIDStatus durch die Teams in der Hochschulambulanz durchgeführt.

\section{Operative Patientenbehandlung}

Hauptaspekte bei der kritischen Indikationsstellung zu HNO-ärztlichen Operationen sind zum einen der bestmögliche Schutz der Mitarbeiter und zum anderen die Einsparung von benötigten Ressourcen i. S. v. Material und Arbeitskräften. Daher wird bei allen Patienten mit OP-Indikation ein Abstrich auf SARS-CoV-2 abgenommen. Die zusätzliche Belastung des Labors war angesichts der initial noch stärker limitierten Testkapazitäten mit der Laborleitung abgestimmt worden. Die Dringlichkeit einer Testung wird auf dem Anforderungsschein ausgewiesen.

Anschließend erfolgt zur Reduktion der Anzahl der Eingriffe das weitere Vorgehen in Abhängigkeit von der Dringlichkeit des chirurgischen Eingriffs entsprechend des in $\mathbf{A b b} \mathbf{5}$ dargestellten Schemas. Wenn möglich, wird auf eine operative Maßnahme ohne negativen Abstrich verzichtet. Bei Eileingriffen wird das Testergebnis abgewartet. Bei positivem Resultat erfolgt der Eingriff unter maximalem Infektionsschutz im COVID-positiven Bereich des Universitätsklinikums. Bei dringlichen OP-Indikationen mit einem Zeithorizont von 2-3 Wochen und nachgewiesener Infektion mit SARS-CoV-2 wird, wenn möglich, zunächst der COVIDKrankheitsverlauf abgewartet. Bei asymptomatischem Verlauf kann der Patient nach negativem Abstrichergebnis und Beendi- 


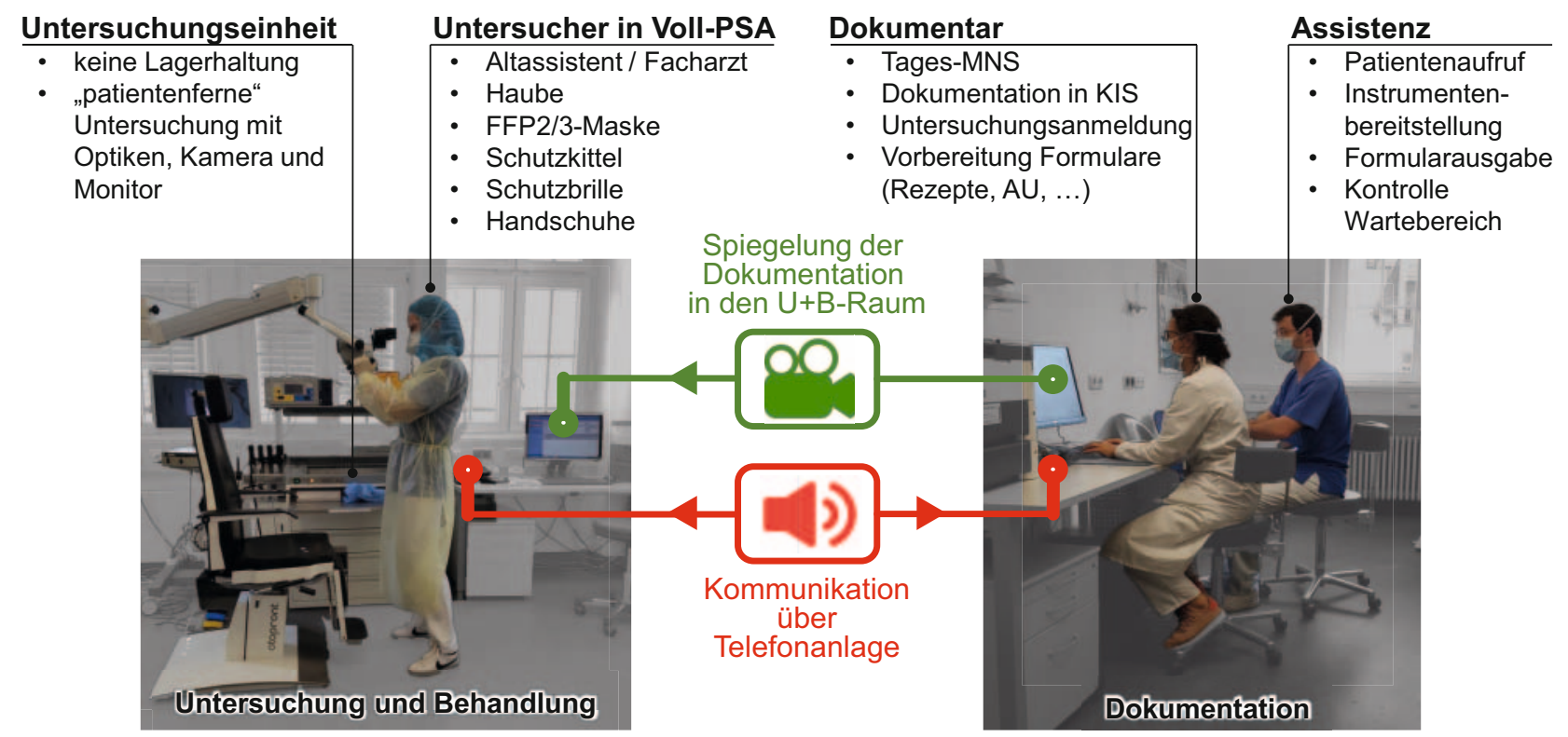

- Abb.4 Aufbau und Funktionszuweisung innerhalb eines Untersuchungsteams. Der Untersucher wird von allen Dokumentations- und Organisationsaufgaben entbunden, um eine möglichst kurze Kontaktzeit zum Patienten zu gewährleisten. Eine knappe Anamnese und die symptombezogenen Untersuchungsbefunde werden über die Audiokommunikation zum Dokumentar übertragen und in das KIS eingepflegt. Die Patientenakte wird auf den Monitor des Untersuchers gespiegelt, der die Eintragungen kontrollieren kann.

gung der Heimquarantäne unter regulären Kautelen in der HNOKlinik behandelt werden. Bei symptomatischem Verlauf wird der Patient in den COVID-positiven Bereich des Universitätsklinikums aufgenommen und von der HNO dort betreut.

Indikationen zu chirurgischen Maßnahmen sollten bei positivem SARS-CoV-2-Nachweis sehr kritisch abgewogen und nur bei zwingender Notwendigkeit durchgeführt werden. Aufgrund der potenziellen Gefährdung der Behandler im OP-Saal wird in unserem Betriebskonzept zwischen Eingriffen mit normalem Infektionsrisiko (Weichteileingriffe) und Hochrisikoeingriffen mit potenzieller Aerosolbildung (alle Eingriffe an den oberen Luftwegen, insbesondere in bzw. durch Nase und Nasenrachen, Versorgung von Nasenblutungen, Tracheotomie) unterschieden.

Bei planbaren Operationen werden Hochrisikoeingriffe (Nase/ Nasenrachen) Eingriffen mit niedrigem Risiko nachgestellt. Der Eingriff mit dem höchsten Risiko bestimmt die Wahl des durch die Mitarbeiter im Operationsbereich zu tragenden Tages-MNS (chirurgischer MNS, FFP2- oder FFP3-Maske). Ein MNS wird gemäß aktuellem Hygieneplan von einem Mitarbeiter im OP-Bereich für den gesamten Tag getragen (außer bei Durchfeuchtung, Kontamination oder Beschädigung). Die Anwendung aerosolbildender Techniken (Laser, Bohrer, Shaver, Koagulation) wird auf das absolut erforderliche Minimum begrenzt. Personalwechsel während der Eingriffe werden zwecks Einsparung von PSA vermieden. Diese Maßnahmen gelten für alle im OP-Saal tätigen Berufsgruppen.

\section{Umsetzungsphase}

Das oben dargestellte Betriebskonzept konnte in seinen wesentlichen Zügen innerhalb von 48 Stunden umgesetzt werden. Die
Patientenbehandlung in Untersuchungsteams ließ sich durch ausführliche Kommunikation und die hohe Einsatzbereitschaft aller Beteiligten ohne wesentliche Einschränkungen umsetzen. Relevante Wartezeiten oder Menschenansammlungen im Wartebereich konnten hierdurch vermieden werden. Zusätzliche bauliche Maßnahmen (Hinweisschilder, Wegeführung, Glaswände zum Schutz vor Tröpfcheninfektion im Tresenbereich etc.) wurden im Laufe der folgenden Tage angepasst. Eine abschließende VorOrt-Begehung durch die hygienebeauftragten Ärzte, die Hygienefachkräfte und den Krankenhaushygieniker bestätigte die Praktikabilität und die Erfüllung der Hygieneempfehlungen, sodass die beschriebenen Maßnahmen „bis auf Weiteres“ als aktuell gültiges Betriebskonzept implementiert wurden.

\section{Diskussion}

Die SARS-CoV-2-Pandemie stellt alle Gesundheitseinrichtungen vor besondere Herausforderungen. Besonders Einrichtungen der hochschulmedizinischen Maximalversorgung müssen sich auf die Behandlung potenziell infektiöser Patienten und nachgewiesener COVID-19-Fälle vorbereiten. Um den veränderten Erfordernissen der Hygienevorgaben, des Arbeitsschutzes und der klinischen Behandlungsprozesse gerecht zu werden, wurde eine kurzfristige Entwicklung und Implementierung eines auf die neuen Vorgaben angepassten Betriebskonzeptes erforderlich.

Vorrangiges Ziel des hier vorgestellten Konzeptes war die Realisierung eines optimalen Mitarbeiterschutzes mit den gegebenen Ressourcen (Zeit bis zur Erstellung, bauliche Infrastruktur, Verfügbarkeit von Verbrauchsmitteln und Schutzausrüstung, Anzahl der 


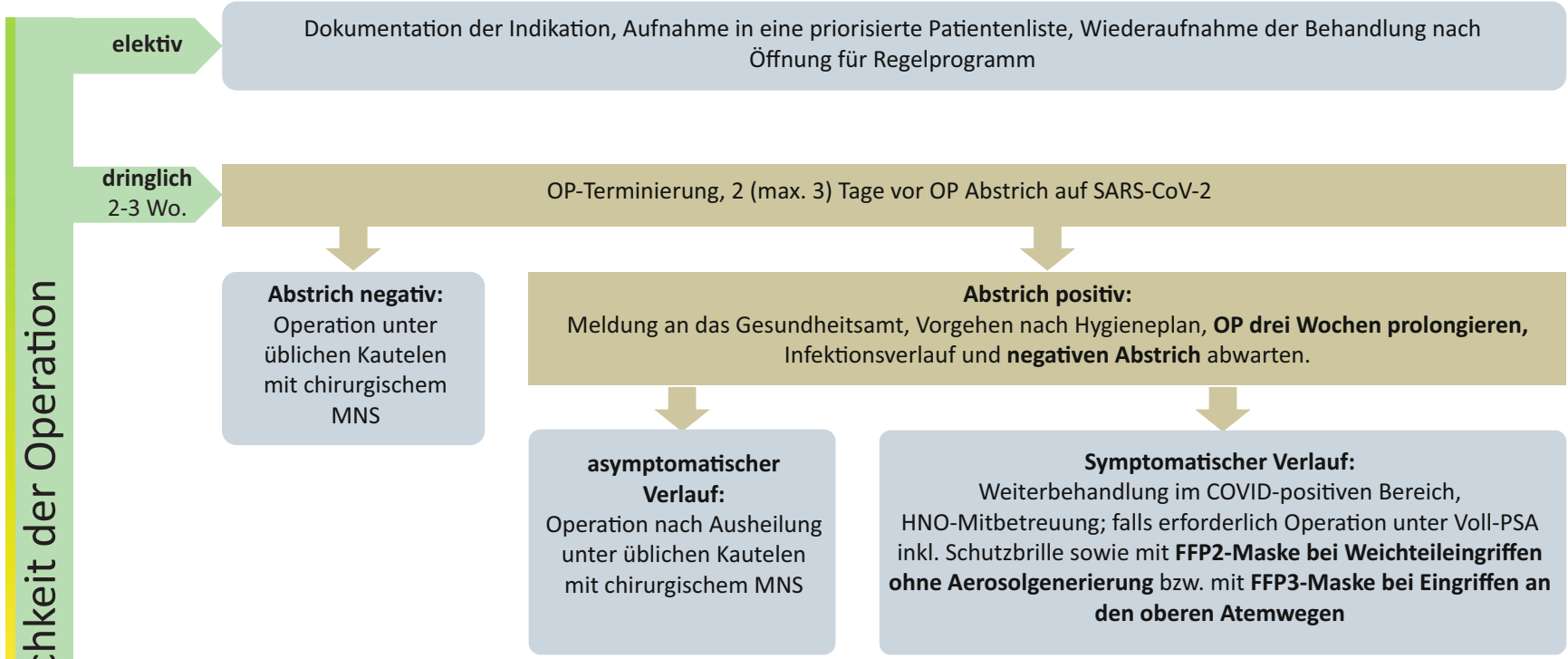

Eilfall Abstrichnahme sofort, Unterbringung in „grauer Zone“ auf Station, weiteres Vorgehen nach SARS-CoV-2-Abstrichergebnis:

$6-24 h$

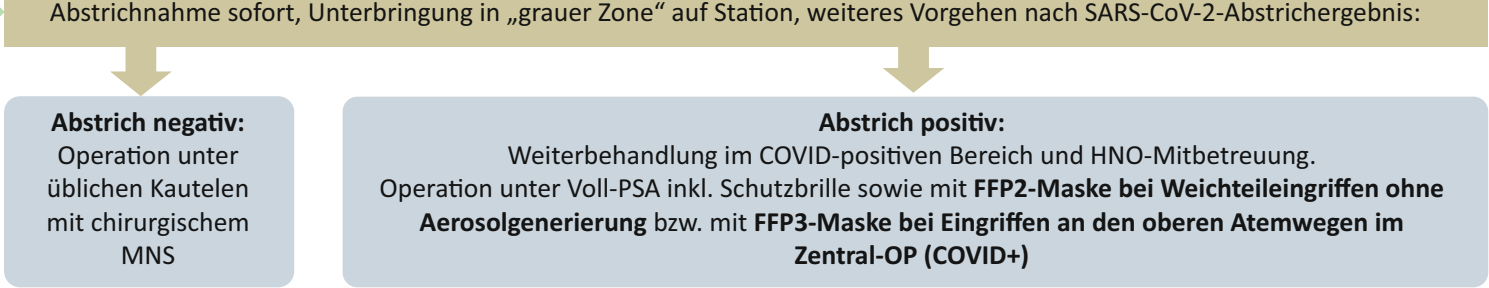

Abstrichnahme sofort, Operation unter Voll-PSA inkl. Schutzbrille sowie mit FFP2-Maske bei Weichteileingriffen ohne Aerosolgenerierung bzw. mit FFP3-Maske bei Eingriffen an den oberen Atemwegen. Postoperativ Zimmerisolation auf Station mit max. 1 Mitpatient (zur Minimierung der Kontakter).

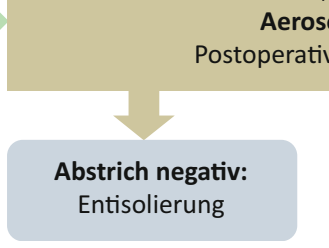

- Abb.5 Flussschema der operativen Patientenbehandlung. Entscheidung über die entsprechende Vorgehensweise nach Dringlichkeit der Operation (grün). Operationen mit unklarem COVID-Status sollten vermieden werden. Bei unumgänglichen Operationen und COVID-positiven Patienten Durchführung des Eingriffs im COVID-positiven Bereich unter Maximalschutz.

Mitarbeiter). Um dieses Ziel zu erreichen, wurden die bestehenden Arbeitsprozesse unter Berücksichtigung des internen Hygieneplans, der aktuellen Empfehlungen von Fachgesellschaften und des Robert Koch-Instituts umfangreich operationalisiert und - wenn notwendig - angepasst. Ein zentrales Element stellte dabei die Reduktion der Anzahl und der Dauer der Patientenkontakte dar.

Der angestrebte Mitarbeiterschutz dient sowohl dem Eigenschutz als auch einer bestmöglichen Sicherstellung der Betriebsbereitschaft der HNO-Klinik für die Versorgung von Notfällen und lebensbedrohlichen Erkrankungen im Fachgebiet. Direkt nachgeordnetes Ziel ist die Patientensicherheit. Dies betrifft sowohl den Schutz des Patienten vor Infektion durch Dritte als auch die Wahrung des Facharztstandards für die Patientenbehandlung selbst. Zwischen Mitarbeiter- und Patientenschutz musste ein ausgewogener Kompromiss getroffen werden, da beispielsweise eine ausführlichere Anamnese und umfassendere Untersuchung des Patienten mit einer längeren Expositionszeit zwischen Arzt und Patient einhergeht. Die Deutsche Gesellschaft für Hals-Nasen-Ohren-Heilkunde, Kopf- und Hals-Chirurgie e. V. empfiehlt, die HNO-ärztliche Untersuchung unter strenger Indikationsstellung und konsequent symptombezogen durchzuführen [13].

Das auf dieser Basis entwickelte Betriebskonzept konnte innerhalb weniger Tage erfolgreich umgesetzt werden. Der Prozess wurde durch die Mitarbeiter der Krankenhaushygiene begleitet: Hierzu waren bereits im Vorfeld Schulungen zum fachgerechten An- und Ablegen der PSA durchgeführt worden, da besonders diese Arbeitsschritte mit einem hohen Kontaminationsrisiko verbunden sind. Das Betriebskonzept sah zur Risikominimierung mög- 
lichst wenige Mitarbeiter in Voll-PSA mit fest eingeplanten Wechseln und Pausenzeiten vor. Diese Aspekte sollten auch die Anwendung und den Verbrauch der knappen Ressourcen an PSA positiv beeinflussen.

Bei der praktischen Umsetzung zeigte sich allerdings, dass die Arbeitsbelastung für einen einzelnen Mitarbeiter unter Voll-PSA über einen kompletten Arbeitstag nicht vertretbar ist. Es wurde unter anderem über eine vermehrte Abgeschlagenheit und Gefühlsstörungen im Bereich der Nase durch das mehrstündige Tragen einer FFP-Maske berichtet. Auch aus Italien wurden in der Laienpresse entsprechende Symptome bis hin zu Hautläsionen im Gesichtsbereich durch den Druck der FFP-Masken geschildert [15]. Auch aus arbeitsmedizinischer Sicht ist die Anwendung der FFP-Masken über eine gesamte Schicht nicht vertretbar. Entsprechende Arbeitsschutzregeln der Bundesanstalt für Arbeitsschutz und Arbeitsmedizin sind zu beachten [16]. Deswegen wechseln Untersucher und Dokumentar nach ca. 4 Stunden ihre Funktionen.

Eine erhöhte Arbeitsbelastung der Mitarbeiter wurde auch im Nacht- und Wochenenddienstbetrieb festgestellt. Daher wurde für die üblicherweise besonders arbeitsintensiven Tage (Samstag und Sonntag) eine zusätzliche ärztliche Rufbereitschaft eingerichtet, sodass sich 2 Ärzte wechselseitig ablösen können.

Insgesamt wurden aber die Veränderungen von den Mitarbeitern aller Berufsgruppen durchweg positiv aufgenommen und engagiert umgesetzt. Die Mehrbelastung durch die Patientenuntersuchung in Voll-PSA wurde durch die Reduktion der Patientenzahl teilweise kompensiert und auch aufgrund des erhöhten Sicherheitsempfindens toleriert. Auch seitens der Patienten wurde die Intention der Maßnahmen unmittelbar akzeptiert und den neuen Regelungen und Schutzmaßnahmen großes Verständnis entgegengebracht

\section{Ausbaustufen des Konzeptes}

Das Betriebskonzept unterliegt angesichts der raschen Veränderung der SARS-CoV-2-Pandemie einem dynamischen Anpassungsprozess. Potenziell bestehen zahlreiche Möglichkeiten, den Leistungsumfang, die Infrastruktur oder die Ressourcen an die jeweiligen Erfordernisse anzupassen:

\section{Leistungsumfang}

Entgegen der anfänglichen Annahme, dass die derzeitige Reduktion des üblichen Leistungsumfangs auf wenige Wochen limitiert sein könnte, sind wir aktuell (Stand Ende April 2020) in der Situation, dass inzwischen auch HNO-Patienten mit chronischen Krankheitsverläufen einer Behandlung zugeführt werden müssen, um mögliche Folge- und Langzeitschäden zu vermeiden. Dies betrifft Erkrankungen, die zwar einige Wochen Aufschub ermöglichen (z. B. Tumornachsorgen, chronisch-entzündliche Geschehen, Hörstörungen mit Kommunikationsproblemen), aber nicht unbegrenzt prolongierbar sind, ohne das langfristige Patientenwohl negativ zu beeinflussen. Aus diesem Grund wurde zusätzlich eine Telefon- bzw. Videosprechstunde etabliert und in das Betriebskonzept übernommen. Das Ziel bestand darin, zu uns bekannten Patienten Kontakt zu halten, den aktuellen Gesundheitsstatus zu erfragen und die adäquate Weiterbetreuung zu gewährleisten.
Seit dem Inkrafttreten der Reduktion am 16.03.2020 haben sich mehrere hundert Behandlungsanfragen angesammelt, die bei einer schrittweisen Ausweitung der klinischen Leistungen sorgfältig nach medizinischer und sozialer Dringlichkeit priorisiert werden müssen. Die Telefon- und Videosprechstunde könnte im Übergang zum Regelbetrieb hierbei ein wertvolles Instrument sein.

\section{Infrastruktur}

Das derzeitig umgesetzte Betriebskonzept wurde unter der Annahme eines konstanten bzw. limitierten Patientenaufkommens erstellt. Im Fall eines deutlich erhöhten Patientenandrangs bzw. steigender Durchseuchung der Bevölkerung könnte eine Anpassung des Konzeptes im Hinblick auf die Patientenführung innerhalb der HNO-Klinik erforderlich sein. Hierzu würden dann optional die Patientenannahme und eine Doppeltriage auch ausgelagert vor dem Gebäude, z. B. in einem Zelt, möglich sein, um die sensiblen medizinischen Kernbereiche innerhalb des HNOKlinikgebäudes vor einer unkontrollierten Virusexposition zu schützen. Vergleichbare Maßnahmen wurden bereits an anderen universitären Einrichtungen, wie beispielsweise in Köln, durch das Technische Hilfswerk (THW) erfolgreich umgesetzt [17].

\section{Ressourcen}

Eine weitere Eskalationsstufe zur Optimierung des Mitarbeiterschutzes hinsichtlich einer erhöhten Virusexposition könnte die Verwendung von tragbaren, aktiven Luftfilterrespiratoren (powered air-purifying respirator, PAPR) anstelle der Halbmasken darstellen [18]. Da bisher nicht abschließend geklärt ist, inwieweit FFP2/3-Masken einen hinreichenden Schutz bei Hochrisikoeingriffen im Bereich der oberen Atemwege mit relevanter Aerosolbildung bei COVID-positiven Patienten bieten, empfiehlt die US-amerikanische Fachgesellschaft American Academy Of Otolaryngology - Head And Neck Surgery daher, die Anwendung solcher Atemschutzsysteme zu erwägen [19]. Ein PAPR besteht aus einer Vollmaske für den Kopf, in die über einen, oft am Gürtel getragenen Ventilator gefilterte Atemluft zugeführt wird. Eine Beschreibung der Vor- und Nachteile findet sich bei Wong et al. [18]. Zwar haben die PAPR-Systeme einen höheren Sicherheitsfaktor gegenüber den FFP-Masken [20], eine Reduktion der (aerogenen) Virusübertragung konnte bisher aber nicht nachgewiesen werden [21].

\section{Kritische Betrachtung}

Hauptziel bei der Erstellung dieses Betriebskonzeptes war es, die Anzahl und die Dauer des Patientenkontaktes auf ein Minimum zu beschränken, um ein Maximum an Mitarbeiterschutz zu erreichen und gleichzeitig die organisatorischen und strukturellen Voraussetzungen für eine sichere Patientenbehandlung im ambulanten, stationären und operativen Bereich zu schaffen. Hierzu war es notwendig, die Anzahl und die Dauer des individuellen Patientenkontaktes deutlich zu reduzieren. Nach der subjektiven Einschätzung der bei der Erstellung beteiligten Mitarbeiter und nach mehrfacher Prüfung und Begehung durch die Krankenhaushygiene wurden diese Ziele erreicht. Eine objektive Validierung steht zum gegenwärtigen Zeitpunkt noch aus. 
Als ein potenzielles Risiko muss allerdings betrachtet werden, dass derzeit lediglich eine streng symptombezogene Untersuchung der Patienten stattfindet. Dies könnte zur Folge haben, dass relevante Zufallsbefunde nicht erfasst werden. Daraus möglicherweise resultierende Konsequenzen für die Patienten sind denkbar. Gleiches gilt für die derzeit notwendigen Terminverschiebungen neuer Patienten seit Beginn der Corona-Pandemie. Auch hierbei könnten relevante Krankheitsbilder zurückgestellt worden sein. Die enge Anbindung medizinisch unklarer Patienten, z. B. über Videoberatungen oder Telefonsprechstunden, sind daher gegenwärtig besonders nützlich, um diese Risiken zu minimieren. In der aktuellen Situation kommt einer verantwortungsvollen Aufklärung eines Patienten mit dem Hinweis der jederzeit möglichen Wiedervorstellung bei Beschwerdepersistenz oder -zunahme eine besondere Bedeutung zu.

Die verlässliche Erreichbarkeit der HNO-Klinik und die direkte Kommunikation mit niedergelassenen HNO-Kollegen bieten eine weitere Möglichkeit, dringliche Fälle klar zu bahnen und einer kurzfristigen Behandlung zuzuführen.

Die beschriebenen Maßnahmen wurden am 16.03.2020 eingeführt und haben auch zum Zeitpunkt der Fertigstellung des vorliegenden Manuskripts (Ende April 2020) weiterhin Gültigkeit. Insgesamt handelt es sich bei der Erstellung eines Betriebskonzeptes besonders in der Zeit einer Pandemie um einen hochdynamischen Prozess, der immer wieder an den aktuellen Erkenntnisstand angepasst werden muss. Eine regelmäßige Überprüfung der Gültigkeit bereits veranlasster Maßnahmen ist daher wesentlicher Bestandteil des Konzeptes. So wurde zwischenzeitlich das Betriebskonzept weiter aktualisiert und inzwischen das verbindliche Tragen des MNS auch bei Patienten zum Selbst- und Fremdschutz eingeführt. Auch wenn viele Voraussetzungen eines Betriebskonzeptes (Bau, Struktur, Personal etc.) standortspezifisch sind, möchten wir mit dieser Arbeit unsere Erfahrungen teilen und eine Umsetzungsmöglichkeit darstellen. Besonders wichtig ist uns der Hinweis, dass eine strukturierte, interdisziplinäre Vorbereitung es ermöglicht, in kurzer Zeit sowohl für die Patienten als auch für die Mitarbeiterinnen und Mitarbeiter adäquate Problemlösungen zu entwickeln.

\section{Interessenkonflikt}

Die Autorinnen/Autoren geben an, dass kein Interessenkonflikt besteht.

\section{Literatur}

[1] Mackenzie JS, Smith DW. COVID-19: a novel zoonotic disease caused by a coronavirus from China: what we know and what we don't. Microbiol Aust. published online ahead of print 2020 Mar 17

[2] Zhu N, Zhang D, Wang W et al. A novel coronavirus from patients with pneumonia in China. N Engl J Med 2019; 382 (8): 727-733

[3] World Health Organization. Coronavirus disease 2019 (COVID-19) Situation Report - 51. [Online] 11. 032020 https://www.who.int/docs/ default-source/coronaviruse/situation-reports/20200311-sitrep-51covid-19.pdf?sfvrsn=1ba62e57_10
[4] Zou L, Ruan F, Huang M et al. SARS-CoV-2 Viral Load in Upper Respiratory Specimens of Infected Patients. N Engl J Med 2020; 382 (12): 11771179

[5] Kim ES, Chin BS, Kang CK et al. Clinical Course and Outcomes of Patients with Severe Acute Respiratory Syndrome Coronavirus 2 Infection: a Preliminary Report of the First 28 Patients from the Korean Cohort Study on COVID-19. J Korean Med Sci 2020; 35: e142

[6] Judson SD, Munster V]. Nosocomial Transmission of Emerging Viruses via Aerosol-Generating Medical Procedures. Viruses 2019; 11 (10): E940. doi:10.3390/v11100940

[7] van Doremalen N, Bushmaker T, Morris DH et al. Aerosol and Surface Stability of SARS-CoV-2 as Compared with SARS-CoV-1. N Engl J Med 2020. doi:10.1056/NEJMc200497

[8] Workman AD, Welling DB, Carter BS et al. Endonasal instrumentation and aerosolization risk in the era of COVID-19: simulation, literature review, and proposed mitigation strategies. Int Forum Allergy Rhinol. Epub ahead of print 2020

[9] Wu Z, McGoogan JM. Characteristics of and Important Lessons From the Coronavirus Disease 2019 (COVID-19) Outbreak in China: Summary of a Report of 72314 Cases From the Chinese Center for Disease Control and Prevention. JAMA. EPub 2020

[10] Robert Koch-Institut. [Online]. https://www.rki.de/DE/Content/InfAZ/ N/Neuartiges_Coronavirus/Situationsberichte/2020-04-16-de.pdf; jsessionid=E0865D9F28075CBDC34E301C7767DCD1.internet121? _blob=publicationFile

[11] Luers JC, Klußmann J, Guntinas-Lichius O. Die Covid-19-Pandemie und das HNO-Fachgebiet: Worauf kommt es aktuell an? Laryngo-RhinoOtologie 2020. doi:10.1055/a-1095-2344

[12] Robert Koch-Institut. [Online]. https://www.rki.de/DE/Content/InfAZ/ N/Neuartiges_Coronavirus/Getrennte_Patientenversorgung.html

[13] Deutsche Gesellschaft für Hals-Nasen-Ohrenheilkunde, Kopf-und HalsChirurgie e.V. [Online]. https://cdn.hno.org/media/Corona-Ticker/ Pragmatischer_Personalschutz_Info_DGHNO_BVHNO_30-03-20.pdf

[14] Robert Koch-Institut. [Online]. https://www.rki.de/DE/Content/InfAZ/ N/Neuartiges_Coronavirus/Massnahmen_Verdachtsfall_Infografik_ DINA3.pdf?_blob=publicationFile

[15] t-online.de. https://www.t-online.de/nachrichten/panorama/ id_87573842/coronavirus-so-sehen-aerzte-nach-13-stundenkrankenhaus-schicht-aus.html. [Online] 19. April 2020

[16] Bundesanstalt für Arbeitsschutz und Arbeitsmedizin. [Online]. https:// www.baua.de/DE/Themen/Arbeitsgestaltung-im-Betrieb/Biostoffe/ $\mathrm{FAQ} / \mathrm{pdf} /$ Empfehlungen-organisatorische-Massnahmen.pdf?_blob= publicationFile $\& \mathrm{v}=9$

[17] Technisches Hilfswerk. Technisches Hilfswerk. [Online]. https://www. thw.de/SharedDocs/Meldungen/DE/Einsaetze/national/2020/corona/ meldung_005_corona_einsatz_3103.html?noMobile $=1$

[18] Wong J, Goh QY, Tan Z et al. Preparing for a COVID-19 pandemic: a review of operating room outbreak response measures in a large tertiary hospital in Singapore. Can J Anesth/J Can Anesth 2020 doi:https://doi.org/10.1007/s12630-020-01620-9

[19] American Academy of Otolaryngology-Head And Neck Surgery. [Online]. https://www.entnet.org/content/academy-supports-cmsoffers-specific-nasal-policy-1

[20] Institute of Medicine Board on Health Sciences Policy. Defining PAPRs and Current Standards. s. I.: DC: National Academies Press (US) 2015

[21] Wax RS, Christian MD. Practical recommendations for critical care and anesthesiology teams caring for novel coronavirus (2019-nCoV) patients. Can J Anesth 2020. doi:https://doi.org/10.1007/s12630-020-01591-x 